\title{
Propuesta de seguimiento al Consejo Técnico Escolar en Educación Básica
}

\author{
Follow-up proposal to the Technical School Council in Basic Education
}

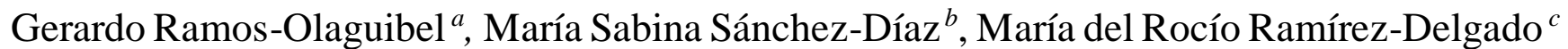
Abstract:

In recent times, in accordance with current educational policy, a relevant role has been assigned to the Technical School Cou ncils, as collegiate bodies that can promote academic management in schools and enhance the reflective work of their teachers. The inte $n$ tion is to support the renewal and change generated by its own actors, despite the diversity of settings in which their practices are developed and developed. This represents an important space for observation and analysis to know the processes followed and, as far as poss ible, provide knowledge so that the external instances of management, supervision, advice can provide the necessary support in said processes. For this reason, the present study is proposed, based on the methodology of evaluative research, as an approach that allows to analyze the processes, practices and policies, in this case, applied in the educational field. This, in order to have the basis for proposing specific topics, content, strategies and activities that are increasingly relevant and closer to the needs of schools.
\end{abstract}

\section{Keywords:}

School Technical Council, collegiate entity Evaluative research

\section{Resumen:}

En los tiempos recientes, de acuerdo con la política educativa actual, se ha asignado un papel relevante a los Consejos Técnicos Escolares, como órganos colegiados que pueden impulsar la gestión académica en las escuelas y potenciar el trabajo reflexivo de sus docentes. La intención es apoyar la renovación y el cambio que generan sus propios actores, a pesar de la diversidad de escenarios en los que se desarrollan y gestan sus prácticas. Lo que representa un espacio importante de observación y análisis para conocer los procesos seguidos y, en la medida de lo posible, aportar conocimiento para que las instancias externas de dirección, de supervisión, asesoría puedan otorgar los apoyos necesarios en dichos procesos. Por ello se propone el presente estudio basado en la metodología de la investigación evaluativa, como el enfoque que permite analizar los procesos, prácticas y políticas, en este caso, aplicadas en el ámbito educativo. Esto, con la finalidad de contar con bases para proponer temas, contenidos, estrategias y actividades específicas, que sean cada vez más pertinentes y cercanos a las necesidades de las escuelas.

\section{Palabras Clave:}

Consejo Técnico Escolar, órgano colegiado, investigación evaluativa

\section{Introducción}

Las diversas políticas educativas que se han implementado en nuestro país, a lo largo de varias décadas, han buscado el establecimiento de la mejora, con enfoques diversos, a veces centrados en acercar a la escuela decisiones académicas derivadas de expertos externos 0 en otros momentos, obedeciendo a movimientos internacionales, donde sólo se aplican y se siguen estrategias definidas.

Sin embargo, en los tiempos actuales se ha observado que los mejores resultados se obtienen cuando los colectivos docentes definen el rumbo del cambio y la manera de renovarse a partir de reconocer sus

\footnotetext{
a Autor de Correspondencia, Universidad Pedagógica Nacional, https://orcid.org/0000-0003-4620-1329,

Email: grolaguibel@hotmail.com.com

b Centro de Asistencia Técnica a la Escuela y Formación Docente “María Lavalle Urbina”, https://orcid.org/0000-0002-1630-9438, Email: sabinasanchez0717@gmail.com
}

c Centro de Asistencia Técnica a la Escuela y Formación Docente "Rosario Gutiérrez Eskildsen”, https://orcid.org/0000-0002-8877-4208, Email: rociodiplos@gmail.com 
necesidades, del diálogo, de la intervención activa y reflexiva de los maestros al interior de cada plantel educativo. Es decir, cuando se reconocen como actores importantes para la mejora y son ellos los que lo lideran, mediante sus acciones a favor de su propia formación, que a su vez, incide en la de las niñas, niños y adolescentes (NNA)'.

Realizar este estudio de seguimiento, permite acercamos a las escuelas y reconocer los procesos que en ellas se generan, la forma como cada colectivo docente los construye bajo sus propios ritmos y recursos. En este sentido cobra particular relevancia el acompañarlos e intervenir sin alterar sus decisiones. Por tanto, su stentar el estudio en la metodología basada en el enfoque de la investigación evaluativa que se caracteriza por desarrollar un proceso sistemático e intencional, que recupera información valiosa, valida y fiable, orien tada a valorar procesos "... para la toma de decisiones, para la gestión y la planificación, la solución de problemas y la construcción práctica de programas" (Escudero 2011).

En el caso particular del estudio, la valoración se en foca en los procesos que se siguen en el desarrollo del Consejo Técnico Escolar (CTE), para que posteriormente, se tomen decisiones de mejora pertinentes por parte de las instancias de dirección, supervisión, asesoría y apoyo cercanas a las escuelas y, de modo indirecto, del campo social en el que se encuentran inmersas éstas.

Con tal propósito, planteamos las siguientes preguntas de investigación: ¿Cómo incide la gestión del cocimiento en la práctica profesional de los docentes y de éstos, en el aprendizaje del alumnado? ¿Cómo ayuda la dinámica de trabajo generada por los docentes y directivos en el CTE, para la construcción de los procesos de autonomía de gestión académica de cada centro escolar?

El objetivo general del estudio es: Conformar al Con sejo Técnico Escolar (CTE) como espacio académico que oriente, planee y materialice la mejora del aprendizaje de las niñas, niños y adolescentes.

Los Consejos Técnicos Escolares (CTE) son considerados como el órgano de mayor decisión técn ico pedagógica, y en sí mismos representan una estrategia que pretende la formación integral del estudiantado y "Ia mejora continua del servicio educativo que presta la escuela, para garantizar que ninguna niña, niño y adolescente se quede atrás o se quede fuera de los aprendizajes y de las oportunidades de desarrollo integral que brinda la vida escolar" SEP (2019:3). Desarrollar un estudio de seguimiento a los CTE, permitirá acercarnos de manera paulatina, a la din ámica que se establece en estos espacios académicos; y como lo plantea, Santizo citada por Bazán (2013), al "trabajo en equipo, el trabajo colegiado y a la colaboración como elementos constitutivos de la Gestión del Conocimiento (GC)".

La investigación evaluativaii, se desarrolla como un proceso donde intervienen diferentes fases, mismas que a continuación se presentan mediante un esquema.

Figura 1. Fases de la investigación evaluativa

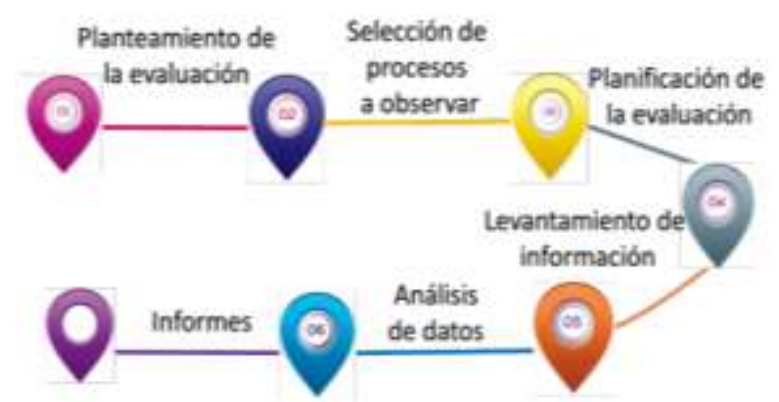

Fuente: Elaboración propia

Estas fases se explicarán atendiendo a los tiempos y espacios que las propias escuelas tienen para su implementación.

La organización del trabajo a desarrollar en los CTE, se centra en dos líneas:

- La construcción del estudio de seguimiento a los CTE en las escuelas de Educación Básica y,

- La autoformación del equipo de observadores (constituido en su mayoría por Asesores técnico pedagógicos, directivos, supervisores, entre otros).

\section{Planteamiento de la evaluación ¿Quién solicita la evaluación?}

Durante los últimos años se ha realizado, como una de las acciones que desarrollan autoridades educativas administrativas y directivas, el seguimiento al desarrollo de los Consejos Técnicos Escolares (CTE), tanto a nivel estatal como nacional. En el caso de la estrategia nacional, ha correspondido a la Subsecretaría de Educación Básica, generar la información e instrumentos para el seguimiento a los CTE y abrir el sitio para recabar la información correspondiente, a través de instrumentos como una guía de observación, cuestionarios a docentes y directores de escuela. Asimismo, realizan el análisis de la información recabada y elaboran un informe gráfico para presentar los resultados.

En lo que corresponde al seguimiento estatal, en general, se ha construido a partir de establecer indicadores que sólo dan cuenta del uso de la guía de CTE, sin una perspectiva de focalización correspondiente a su desarrollo, sus acciones, sus procesos autogestivos, las problemáticas que enfrentan y las posibilidades que 
tienen desde una perspectiva académica, de la mejora de los aprendizajes de los edu candos.

En el caso de los CTE, en la actualidad es considerado, de manera formal, como el "órgano colegiado de mayor decisión técnico pedagógica, que tiene como propósito principal la mejora continua del servicio educativo que presta la escuela, para garantizar que ningún niña, niño o adolescente se quede atrás o se quede afuera de los aprendizajes y de las oportunidades de desarrollo integral que brinda la vida escolar", como lo señala el documento de Organización de los Consejos Técnicos Escolares (SEP, 2019: 3).

Sin embargo y debido al poco tiempo de reformulación, son pocas las investigaciones que se tienen hasta la fecha, sobre el impacto académico en las escuelas de educación básica.

El presente estudio de seguimiento al Consejo Técnico Escolar, sustentado en el enfoque de la investigación evaluativa, es entendido, como un proceso en sí mismo, riguroso, controlado y sistemático de recogida y análisis de información válida y fiable para tomar decisiones colegiadas, elemento que coincide con el sentido actual del CTE, mismo que propone diversas acciones en los procesos de gestión, académicos y de operación, que serán objeto del seguimiento.

En este sentido, se busca conocer lo que acontece al interior de los colectivos docentes para identificar sus problemáticas, reconocer los obstáculos a los que se enfrentan y cómo éstos limitan o posibilitan, desde un enfoque académico, la mejora del aprendizaje del alumnado de cada plantel educativo. El desarrollo de este trabajo permitirá obtener resultados para la intervención en la toma de decisiones y fortalecer así, a los colectivos docentes desde una perspectiva académica y de reflexión colectiva, que oriente la selección de ofertas académicas acorde a sus necesidades.

\section{¿Para qué se solicita?}

Los Consejos Técnicos Escolares, son una estrategia gubernamental de política educativa cuyo propósito es generar "acciones de mejora escolar en beneficio de sus estudiantes", para ello, los desafíos y oportu nidades que se tienen en el marco de la Nueva Escuela Mexicana son:

- "Promover el sentido de comunidad, sin exclusión alguna, con mayor participación de las NNA y sus familias, en la mejora escolar.

- Establecer un Programa Escolar de Mejora Continua (PEMC), que coloque a las NNA al centro del quehacer educativo, que integre una visión compartida de toda la comunidad escolar, con objetivos de alcance multianual y ámbitos de acción que consideren distintas con diciones de la realidad escolar.

- Favorecer el diseño e implementación paulatina de buenas prácticas docentes, en la escuela y en las aulas, con énfasis en la equidad, excelencia, inclusión, integralidad, participación democrática y la cultura de paz.

- Dar espacio al colectivo docente para abordar temas de su particular interés o preocupación, de acuerdo con sus circunstancias y contexto específico. Los docentes no disponen de otro espacio de tiempo para reflexionar, organizarse y tomar decisiones acerca de la escuela" (SEP, 2019).

Como parte de la nueva organización de los CTE, desde el ciclo escolar 2019-2020, se ha planteado como desafío, organizar las sesiones de estos, mediante tres momentos de trabajo, con tiempos definidos para cada momento (SEP, 2019a: 4).

Figura 2. Momento de trabajo de los Consejos Técnicos Escolares

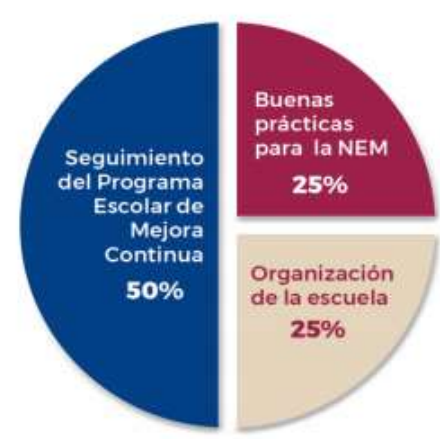

Fuente: Elaboración propia

El esquema permite visualizar entre otros aspectos, la intención académica prevista desde el "deber ser", para el desarrollo de los CTE, lo que lleva a reconocer si esta intención se concreta en la cotidianeidad de su operación, a través de la orientación, planeación y materialización de la mejora en el aprendizaje del alumnado, así como el tipo de problemáticas que están atendiendo y las acciones 0 actividades que implementan, para incidir en las mismas en los NNA y comunidad escolar en general.

Desarrollar un estudio de seguimiento a los CTE, permitirá acercarnos de manera paulatina, a la dinámica que se establece en estos espacios académicos; y como lo plantea, Santizo, citado por Bazán (2013), el trabajo en equipo, el trabajo colegiado y la colaboración son los elementos constitutivos de la gestión del conocimiento. El análisis de lo que acontece en estos espacios (procesos), en específico los generados en los CTE, abre múltiples posibilidades de reconocer los diversos escenarios y las dinámicas en que se desarrollan sus 
propuestas de trabajo, para identificar las innovaciones que se hacen mediante el intercambio entre escuelas y los tipos de diálogo que se establecen, con la finalidad de mejorar los aprendizajes de los estudiantes; el desarrollo de las habilidades profesionales de los docentes, para avanzar en la mejora y la transformación de su práctica, además de la incidencia que estos procesos tienen en la au tonomía de gestión de cada centro escolar.

\section{¿Qué se pretende evaluar?}

En los tiempos recientes se busca una revitalización de las funciones de los CTE, a través del fortalecimiento de las actividades pedagógicas en el conjunto de las acciones que conforman el hacer cotidiano de las escuelas. Es así que se espera centrar el trabajo en la enseñanza como tarea prioritaria de la escuela y de los docentes, vinculado al análisis de los resultados educativos que obtienen los alumnos, la mejora de la práctica del docente, la innovación y gestión del conocimiento. Es importante reconocer que, los retos que tienen los colectivos docentes y directivos son diversos, para que de manera real se concrete el "deber ser", es decir la tarea para la que fueron creados los Consejos Técnicos Escolares.

Para efectos de este artículo solo abordaremos la primera línea de investigación: La construcción del estudio de seguimiento a los CTE en las escuelas de Educación Básica, en donde proponemos cinco líneas de análisis mismas que describimos a continuación:

Figura 3. Líneas de análisis para el seguimiento a los CTE

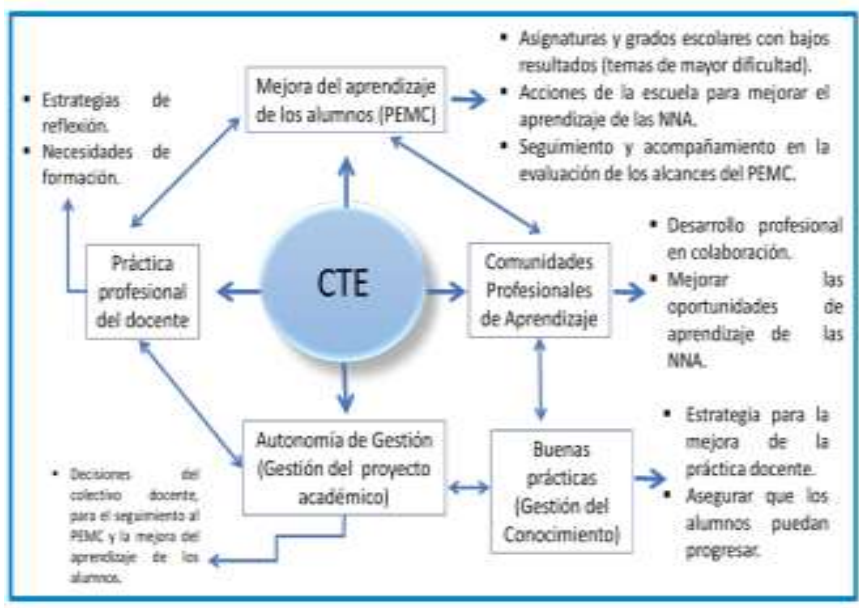

Fuente: Elaboración propia

\section{Mejora del aprendizaje de los alumnos:}

Como lo señala Francesc, Pedró (2005:12,14), “Un plan de mejora, es la propuesta de actuaciones, resultante de un proceso previo de diagnóstico, que recoge y formaliza los objetivos de mejora y las correspondientes actuaciones dirigidas a fortalecer los puntos fuertes y resolver los débiles, de manera priorizada y temporalizada [...] no es un método de dirección y gestión cerrado y prefijado, sino que facilita variaciones y adaptaciones, con el fin de hacer de la gestión del día a día un instrumento útil y eficaz para el logro de los objetivos fijados y para mantener la razón de ser de la organización".

En la política educativa actual, sobre todo la que está dirigida a la gestión del sistema educativo, aterrizada a la gestión de los centros escolares, se han incorporado categorías de corte teórico pero que se transforman en "instancias político administrativas" que bien se pueden transformar en objeto de estudio en esta propuesta de seguimiento, tales como "comunidades profesionales de apren dizaje", "buenas prácticas", "autonomía de gestión" o "práctica profesional docente"; que bien valen la pena identificar cómo se entienden e instrumentan desde el quehacer educativo de la escuela.

\section{Comunidades Profesionales de Aprendizaje (CPA)}

Richard Dufour, lo concibe "como un proceso continuo en el cual los educadores trabajan colaborativamente en ciclos recurrentes de indagación colectiva e investigación-acción para obtener resultados que beneficien los estudiantes a los que sirven. Considera necesario el compromiso en procesos de indagación colectiva sobre las mejores prácticas de enseñanzaaprendizaje, porque se aprende haciendo para hacer diferente. Las CPA permiten trabajar con datos, para poder informar y retroalimentar la práctica, objeto de la reflexión" (DuFour, 2010:11).

Trasciende el ámbito del desarrollo profesional para abordarse como una forma de pensar y vivir la institución escolar en su conjunto. Conectar el aprendizaje docente con un contexto de trabajo colaborativo; en el que se privilegien las relaciones interpersonales sólidas de modo que todo ello logre tener un impacto profundo en el clima y cultura del centro. Busca generar un cambio en la manera como se podría au todefinir un colectivo docente y por lo mismo se buscaría la mejora en su actividad profesional y a su vez en el aprendizaje significativo del alumnado.

\section{Buenas prácticas:}

Encontramos un primer rasgo para su definición. "una buena práctica es una experiencia de carácter innovador que permite solucionar un problema a través de una mejora en el proceso" (Armijo, 2004).

Las buenas prácticas pueden verse como experiencias concretas que son el resultado de factores personales internos; en relación con un conocimiento de cómo hacer algo, y en el que Kidwell (2008:32, citado por Rodríguez), apunta a dos dimensiones: "saber cómo (know how) pero que también es capaz de (be able to), lo que implica además del conocimiento, una habilidad para actuar. 
Conocer qué (know that), se refiere a la conciencia que tiene la persona de que lo que hace tendrá un resultado, definido no solo por lo que dice la teoría, sino por la práctica recurrente y por la observación de ésta".

Una característica de las buenas prácticas, es que permiten construir el conocimiento en colaboración, tanto entre docentes, como entre docentes-alumnos y llegan a tener un impacto a nivel cognitivo, al mejorar los procesos de aprendizaje de ambos.

\section{Autonomía de Gestión:}

La verdadera autonomía de gestión requiere de crear las condiciones para que cada escuela de manera progresiva, "en un largo proceso, pueda "construir" organizativamente su autonomía. La autonomía es siempre relativa, pudiéndose hablar de mayor o menor autonomía, nunca -en el caso de los centros educativosde autonomía total. La realidad actual y las necesidades del sistema educativo, demandan organizaciones escolares más flexibles, capaces de movilizar su propia capacidad interna para dinamizar su funcionamiento y la búsqueda de procesos de mejora e innovación. "Revisando lo que ha sucedido con los nuevos discursos sobre la autonomía, observamos que, en lugar de haber primado una lógica propiamente pedagógica, se ha quedado en un nuevo modo de gestión, que tran sfiere responsabilizando- al centro escolar determinadas competencias, en lugar de haber sido un medio para potenciar la apropiación de mejores realizaciones" (DuruBellat y Meuret, 2001, citado por Bolívar, 2009:6).

\section{Práctica Profesional del docente}

El quehacer docente presenta obstáculos, que surgen desde el propio docente, que, si no es consciente de los conceptos, representaciones, certezas y creencias que posee, tiende a reproducirlos y por lo tanto a enseñar lo que le enseñaron y de la misma forma en como él lo recibió (Díaz, 2003).

Por su parte John Dewey expone que en educación, la reflexión sobre la práctica se presenta asociada a las corrientes de la pedagogía activa, su aportación se centra en las diferencias entre la acción de rutina la cual se realiza apegado a la tradición y, la acción reflexiva, entendida con una naturaleza dinámica, dependiente del contexto y de las circunstancias en que se presenta, enfrentando los problemas educativos buscando soluciones y estableciendo acciones que los lleve a resolverlos (Dewey, 1989).

Sañudo (2005), propone un modelo de asesoría que impacte el proceso personal para transformar la práctica docente en vez de cursos y talleres de actualización.

En este sentido, resulta importante conocer si en el desarrollo del CTE, los docentes logran incorporar momentos y tiempos para la reflexión de su práctica, cuestionando su quehacer cotidiano y la manera en que resuelven los problemas a los que se enfrentan, a través de procesos cada vez más dialógicos, por medio del intercambio con sus iguales.

\section{¿Qué obstáculos pudieran producirse durante la evaluación?}

Para el desarrollo del proyecto, es posible encontrar dificultades al menos de carácter operativo y académico tanto con el equipo de observadores como en los planteles educativos, objeto de observación.

Los obstáculos pueden ser de carácter operativo, de acuerdo con los siguientes aspectos:

- Disminución en el número de observadores por situaciones personales o laborales.

- Disposición y/o cambios de autoridades educativas en las escuelas de observación.

- No contar con el tiempo suficiente para el diseño, aplicación y procesamiento de instrumentos relacionados con el seguimiento.

- Disposición de los observadores al trabajo de seguimiento.

- Retraso en el registro de los instrumentos aplicados durante el proceso de observación.

- Sesgos en la aplicación de instrumentos y en la información solicitada en los mismos, por parte de los observadores.

Académicos:

- Limitado conocimiento de parte del grupo de observadores en la función y objetivo del seguimiento del CTE.

- Apertura de los supervisores y directores para permitir la intervención de los observadores en el CTE, con participaciones situadas y organizadas, de manera corresponsable.

- Registro de interpretaciones o calificaciones personales por parte de los observadores, de lo sucedido en el CTE.

- Participaciones individuales por parte de los observadores, fuera del marco de seguimiento del CTE.

- Elementos insuficientes del perfil académico de los observadores para atender las necesidades de los colectivos docentes.

- Desarrollar el acompañamiento y asesoría académica, por parte de los observadores, desvinculado a los propósitos del PEMC y de la intención de los CTE.

\section{¿Es posible llevar a cabo la evaluación? (Evaluabilidad: Calidad y barreras)}

Es importante señalar que este estudio, requiere de apoyos institu cionales para su desarrollo. Deberá haber 
comunicación y acuerdos con los niveles educativos para el ingreso a las escuelas, con bajos resultados de acuerdo a lo publicado por PLANEA (Plan Nacional para la Evaluación de los Aprendizajes).

Es necesario que las autoridades administrativas educativas compartan la base de datos de las escuelas con bajos resultados académicos en los campos de formación de Lenguaje y Comunicación, así como de Pensamiento matemático. De este grupo de escuelas se seleccionará una muestra (misma que habrá que reformularse por cuestiones de representatividad cualitativa) de aquellos planteles escolares que representen las diferentes modalidades y turnos, para llevar a cabo el seguimiento de CTE.

\section{¿Existe aceptación por parte de los implicados?}

Como parte de las funciones de las autoridades administrativas educativas, corresponde la participación en el seguimiento a los CTE, esta participación puede estar sustentada y situada, en la medida en que los observadores, conozcan el proyecto y se involucren de manera colegiada en el mismo desde su análisis hasta su operación. Por otra parte, para llevar a cabo el seguimiento, se utilizarán medios electrónicos con la intención de recuperar y sistematizar la información de este seguimiento; así como compartir documentos generados y los instrumentos a utilizarse mediante una carpeta en Dropbox y los correos electrónicos institucionales que se tienen en cada instancia, lo que incide en un ahorro de recursos.

\section{Definición del problema a considerar en el seguimiento al proceso del Consejo Técnico Escolar}

Considerando que el trabajo educativo se lleva a cabo en situaciones donde se presentan problemas prácticos, es necesario tomarlo como punto de partida de lo que acontece en el aula o en la escuela. Éste puede surgir a partir de incoherencias o inconsistencias entre lo que se persigue y lo que en la realidad ocurre. Existen varias técnicas para conocer y describir un problema: listado de problemas, el FODA (fortalezas, oportunidades, debilidades y amenazas), Modelo de espina de Ishikawa, entre otros. Para la presente propuesta se utiliza sólo el árbol de problema como una técnica metodológica que nos permite describir el problema, además conocer y comprender la relación entre las causas que están originando el problema y los posibles efectos que se derivan del mismo

Al ser el Consejo Técnico Escolar (CTE), una estrategia empleada para la resolución de problemáticas de las escuelas, resulta importante plantear: ¿Si el CTE es un espacio académico que pretende orientar, planear y materializar la mejora en el aprendizaje del alumnado, por qué se mantienen bajos resultados en el logro educativo? Este se considera el problema central, a partir del cual se identifican sus posibles causas y efectos, a continuación se presenta de manera esquemática un árbol de problemas:

Figura 4. Árbol de problemas de los CTE

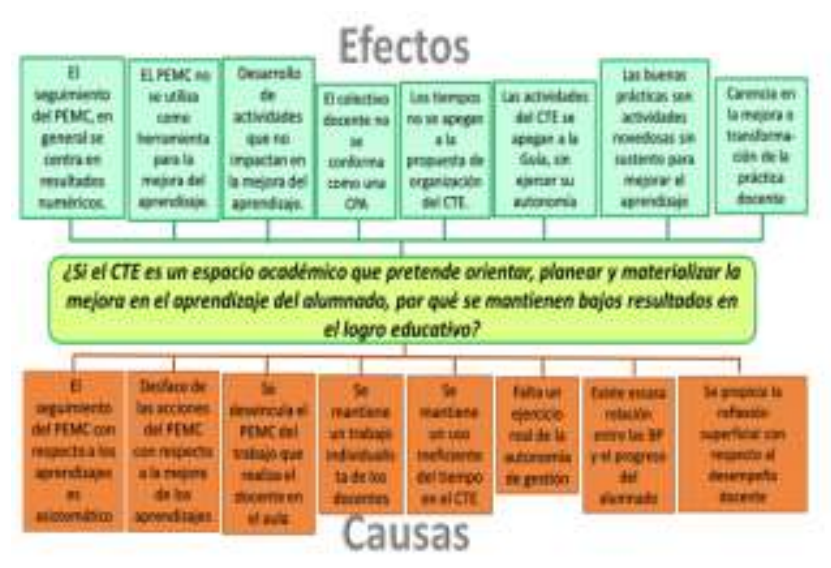

Fuente: Construcción propia.

Con base en este análisis se desglosan algunos objetivos específicos:

- Explicar de manera sustentada las acciones de seguimiento del Programa Escolar de Mejora Continua (PEMC), que permiten valorar el progreso del aprendizaje del alumnado mediante el análisis cualitativo de resultados.

- Discriminar las estrategias de seguimiento de carácter reflexivo que fortalezca la práctica docente y la evaluación del PEMC.

- Distinguir si las estrategias del CTE permiten la formación de Comunidades Profesionales de aprendizaje (CPA).

- Identificar en las buenas prácticas planteadas por las escuelas, las acciones que favorecen la reflexión en el docente y la mejora del aprendizaje de las Niños, Niñas y Adolescentes (NNA).

- Relatar si el tiempo utilizado en el diseño y desarrollo del CTE apoyó a la reflexión y gestión del conocimiento de los docentes y su práctica.

\section{Propuesta}

Como se ha señalado en los apartados anteriores, las líneas de análisis en los que se centrará la observación son:

> Mejora del aprendizaje de los alumnos a través del PEMC o Asignaturas y grados escolares con bajo resultados, obtenidos durante la aplicación 
de PLANEA, contrastados con los resultados que cada escuela recupere y analice en el marco de las sesiones del CTE.

$>$ Acciones implementadas para mejorar el aprendizaje de las NNA.

> Comunidades Profesionales de Aprendizaje:

- Esfuerzos de colaboración que propician el desarrollo profesional del colectivo docente.

- Experiencias de los docentes que permiten mejorar las oportunidades de aprendizaje de las NNA.

- Acciones para favorecer la construcción de la gestión del conocimiento, a través del diálogo profesional de los docentes y directivos.

$>$ Buenas prácticas:

- Estrategias que se aplican en las aulas para la mejora de la práctica docente.

- Compromisos que los colectivos docentes establecen para asegurar que los alumnos puedan progresar y avanzar.

- Problemas y soluciones que se atienden a través de esta estrategia. o Temáticas claves que se abordan.

> Autonomía de Gestión:

- Decisiones que incorporan los colectivos docentes, para el seguimiento al PEMC y la mejora del aprendizaje de los alumnos, en ejercicio de su autonomía.

> Práctica Profesional del docente:

- Estrategias implementadas que propician el diálogo reflexivo, el intercambio de ideas sustentadas, el cuestionamiento de las acciones que realizan y los resultados alcanzados mediante comunidades de aprendizaje y buenas prácticas docentes.

- Necesidades que surgen para contribuir a la formación de los docentes.

Los elementos anteriores, son los aspectos nodales planteados en este estudio, los cuales servirán de guía para la instrumentación y el análisis categorial, definidos a través de:

- Las temáticas planteadas en cada sesión de trabajo, propuestas a través de la Guía del CTE.

- Las formas de organización de los colectivos escolares.

- Los materiales utilizados.

- El logro de los objetivos planteados.

- Los tiempos empleados para cubrir los contenidos.

- La participación de los asistentes.

\section{Planificación de la evaluación (Diseño) (Plan que establece qué unidades van a ser observadas y cuándo)}

Figura 5. Ruta crítica del proceso del estudio:

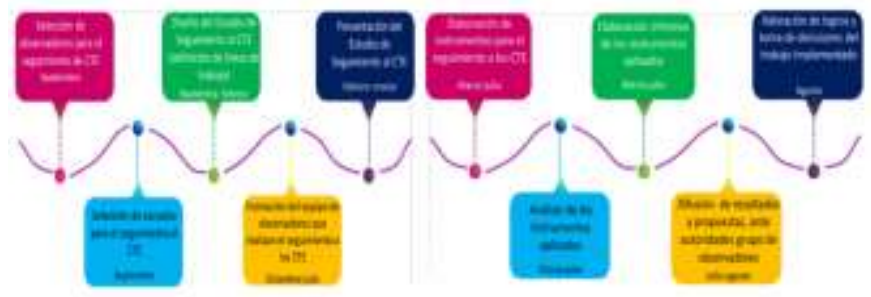

Para su desarrollo, también se incluyen las líneas de análisis para la construcción de los instrumentos de seguimientoiii.

Figura 6. Líneas de análisis para la construcción de los instrumentos

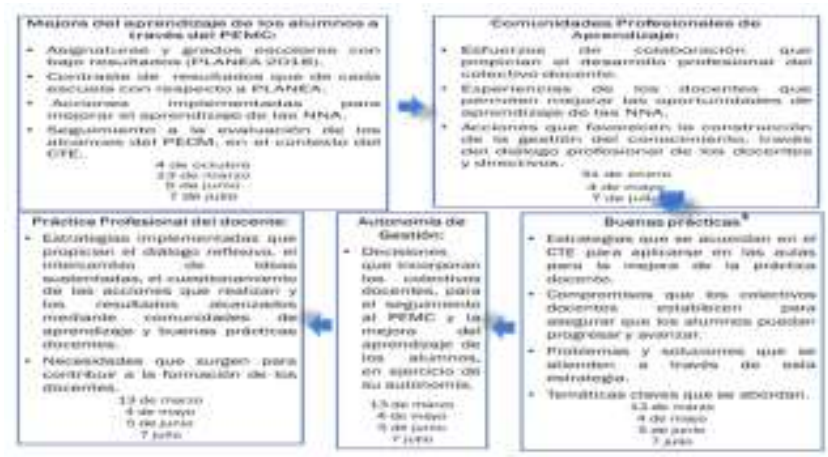

Fuente: Elaboración propia

\section{Levantamiento de información}

Estrategias:

- Autoformación de observadores, mediante reuniones de trabajo colegiado.

- Participación en reuniones preparatorias a cada Consejo Técnico escolar (CTE), tanto de instancias nacionales como de instancias locales.

- Conocimiento de los contenidos de cada sesión del CTE y diseño de instrumentos para la recogida de información. Técnicas e instrumentos necesarios para recopilar información veraz y oportuna. o Se usarán guiones de observación, con indicadores específicos. o Entrevistas guiadas.

- Diarios de campo. Instrumentos (¿Con qué evaluar?): Entrevistas, guías de observación, cuestionarios y bitácoras/diario de campo. Grupos de enfoque. 
Informantes:

Directivos de los niveles educativos y de instancias operativas, supervisores, asesores técnico pedagógicos, directores de escuela y subdirectores, docentes de escuela y personal de CATEyDPD, además de DATEyFD.

Análisis de datos

A partir de la aplicación de los instrumentos, las tareas se centrarán en:

- Recopilación de la información.

- Con base en las categorías de análisis, se organiza la información de corte cualitativo.

- Se procesará estadísticamente, en el caso de información cuantitativa, concentrada en bases de datos y gráficas.

- Se interpretará la información en función de los datos y las preguntas relevantes. Para guiar el análisis, se considerarán los aspectos siguientes:

- Los objetivos planteados y contenidos desarrollados. o Líneas de análisis.

- Las formas de organización y materiales utilizados. o Los tiempos empleados para cubrir los contenidos.

- El tipo y calidad de participación de los asistentes.

- Informes

Temporalidad de los reportes:

- mensual y

- final.

- Contenido de los informes:

- Presentación.

- Contenidos desarrollados en la sesión de trabajo.

- Las formas de organización de los colectivos escolares.

- Descripción del contenido y características de los materiales utilizados.

- El logro de los objetivos planteados.

- Los tiempos empleados para cubrir los contenidos.

- La participación de los asistentes.

- Instrumentos utilizados para la recogida de información 0 Conclusiones y recomendaciones

Difusión:

- Oral

- Escrita

\section{A manera de conclusión}

Es importante conformar un equipo de seguimiento interdisciplinario en que se desarrollen consensos, trabajo colegiado con sus integrantes y se avance en la consolidación académica del mismo, esto con la finalidad de apoyar y fortalecer, sin imponer, el trabajo de los centros escolares y sus colectivos docentes.

Así mismo, el estudiar y analizar lo que se propone desarrollar en las escuelas por parte de las instancias directivas y académicas para diferenciar entre "el deber ser" y lo que ocurre en la operación misma; con la finalidad de documentar aquello que se lleva a cabo en la realidad observada y derivar propuestas pertinentes que permitan el fortalecimiento de los espacios académicos escolares au togestivos encaminados a la mejora real del aprendizaje de las niñas, niños y adolescentes que asisten a los centros educativos en cuestión.

\section{Referencias}

Ariza, Marina (2016). Emociones, afectos y sociología. Diálogos desde la investigación social y la interdisciplina. México: Universidad Nacional Autónoma de México/Instituto de Investigaciones Sociales.

Armijo, Marianela (2004). Buenas Prácticas de la Gestión Pública en América Latina. IX Congreso Internacionaldel CLAD sobre Reforma del Estado y la Administración Pública. 2-5. [En línea]. Madrid España. Disponible en:

https://administracionpublicacomparada.files.wordpress.com/2013/12/ buenas-prc3a1cticas-gestic3b3n-al.pdf [24 de septiembre de 2020].

Bazán, Antonio (2013). El Consejo Técnico Escolar como un espacio para la Gestión del Conocimiento. [En línea]. México. Universidad de Guadalajara. Disponible en: https://rei.iteso.mx/bitstream/handle/11117/5416/Consejo\%20t\% C3\% A9cnico $\% 20$ escolar.pdf?sequence $=4 \&$ isAllowed $=\mathrm{y}$ [24 de septiembre de 2020].

Bolívar, Antonio (2009). La Autonomía en la Gestión como Modelo de Regulación. Revista de Educación. Vol. 19. Universidad Nacional de la Provincia de Buenos Aires.

Bourdieu, Pierre (2000). La dominación masculina. Barcelona: Ana grama.

Capella, Jorge (2005). Módulo para el curso de Gestión del Conocimiento en el campo educativo. Lima: Pontificia Universidad Católica del Perú.

Cornelius, Randolph (1996). The Science of Emotion. Research and Tradition in the Psychology of Emotion. Englewood Cliffs (New Jersey): Prentice-Hall, Inc.

Dewey, John (1989). Cómo pensamos. Cognición y desarrollo humano. Barcelona, España: Paidós.

Díaz, Esther (2003). El sujeto y la verdad II. Paradigmas epistemológicos contemporáneos. En Gorodokin, I. La formación docente y su rela ción con la epistemología. Instituto de Formación Docente Continua San Luis. Escuela Normal Juan Pascual Pringles. Argentina. Universidad Nacionalde San Luis.

DuFour, Richard (2004). What Is a Professional Learning Community? Educational Leadership, 61(8), 6-11. Disponible en http://www.ascd.org/publications/educationalleadership/may04/vol61/ num08/What-Is-a-Professional-Learning-Communityc.aspx. $\left[\begin{array}{ll}20 & \mathrm{de}\end{array}\right.$ abril de 2019]. 
Durkheim, Emile (2008). Las formas elementales de la vida religiosa. Madrid: Alianza Editorial.

Gundermann, Hans (2008). "El método de los estudios de caso" en: María Luisa Tarrés (Coord.) Observar, escuchar y comprender. Sobre la tradición cualitativa en la investigación social, pp. 251-288. México: El Colegio de México.

Jerí, Doris (2008). Buenas prácticas en el ámbito educativo y su orientación a la gestión del conocimiento. Revista Educación. Vol. XVII, N 32, pp. 29-48.

INMUJERES (2015). "Desigualdad en cifras" en Boletín [En línea] Vol. 1, núm. 8. México: Instituto Nacionalde las Mujeres. Disponible en línea en:

http://cedoc.inmujeres.gob.mx/documentos download/BoletinN8.pdf [23 febrero de 2018]

Krook, Mona Lena \& Restrepo, Juliana (2016). "Violencia contra las mujeres en política" en Política y gobierno, Vol. XXIII, núm. 2, pp. 459-490. México: Centro de Investigación y Docencia Económicas.

Micheli, Raphaël(2011). "Las emociones como objetos de construcciones argumentativas". Estudios de Comunicación y Política, núm. 26, pp. 141-166. México: UAM-X.

Pedró, Francesc (2005). Marco general para el establecimiento, el seguimiento y la revisión de los planes de mejora. España. Universidad de Cataluña.

Ritzer, George (2012). Teoría sociológica clásica. México: McGrawHill.

Talamás, Marcela \& Lascurain, Sofía (2016) Protocolo para Atender la Violencia Política Contra las Mujeres. México: Tribunal Electoral del Poder Judicial de la Federación.

Santizo, Claudia (2016). Condiciones Institucionales del trabajo colaborativo como estrategia de cambio del sistema educativo. Perfiles Educativos, Vol. XXXVIII, núm. 153, pp. 154-167. México: IISUEUNAM.

Sañudo, Lya (2005). La formación permanente del profesora do a tra vés de la investigación reflexiva de su práctica Revista Iberoamericana sobre Calidad,Eficiencia y Cambio en Educación.

SEP (2019a). Organización de los Consejos Técnicos Escolares. Ciclo escolar 2019-2020. México. Subsecretaría de Educación Básica.

SEP (2019b). Orientaciones para la organización y realización de las tres sesiones Compartir buenas prácticas. Encuentro entre escuelas. Ciclo escolar 2019-2020. México. Subsecretaría de Educación Básica .

SEP (2019c). Programa Sectorial de Educación 2019-2020. Ciudad de México.

SEP (2019d). Orientaciones para elaborar el programa escolar de mejora Continua. Ciclo escolar 2019-2020. México. Subsecretaría de Educación Básica.

Virgen, Lucy (2013). "17 de octubre de 1953 - Derecho al voto para la mujer en México" en Red Universitaria de Jalisco [En línea]. México: Universidad de Guadalajara. Disponible en: http://www.udg.mx/es/efemerides/17-octubre-0 [21 de diciembre de 2017].

Zavaleta, Ruth (2014). "Cuotas de género, una medida necesaria" en Letras Libres [En línea] núm. 184. Disponible en: http://www.letraslibres.com/mexico/cuotas-genero-una-medidanecesaria

\section{NOTAS}

programas y políticas sociales, en general, y en educación en particular" (Escudero, 2016).

iii En el documento para la organización de los CTE, se especifican fechas exclusivas para la presentación de las Buenas Prácticas durante el intercambio entre escuelas, sin embargo, esta intención no concluye en las sesiones establecidas, continúan en las demás sesiones con la preparación de la experiencia a compartir.

\footnotetext{
${ }^{i}$ A finales de la década de los sesenta nació el movimiento teórico práctico de Mejora de la Escuela (School Improvement), con la idea de que "la escuela debe ser el centro del cambio", con su doble visión: el cambio debe ser liderado por la propia escuela y hay que centrarse en la cultura de la escuela para lograr cambiar la educación (Hargreaves, 1998, citado por Murillo, 2003). Con este movimiento se reconoce que los docentes desde lo individual y como colectivo son elementos clave para la mejora.

ii La investigación evaluativa se ha convertido en las últimas décadas en un enfoque muy importante de actividad investigadora en todos los ámbitos científicos y de manera especial en ámbitos relacionados con los
} 